\title{
A rare case of pericarditis and pleural empyema secondary to transdiaphragmatic extension of pyogenic liver abscess
}

\author{
Eunae Cho ${ }^{1 \dagger}$, Sang Woo Park ${ }^{1 \dagger}$, Chung Hwan Jun ${ }^{1 *} \mathbb{D}$, Sang Soo Shin², Eun Kyu Park ${ }^{3}$, Kyo Seon Lee ${ }^{4}$,
} Seon Young Park', Chang Hwan Park', Hyun Soo Kim', Sung Kyu Choi ${ }^{1}$ and Jong Sun Rew ${ }^{1}$

\begin{abstract}
Background: Transdiaphragmatic extension of pyogenic liver abscess is the rarest cause of pericarditis and pleural empyema. It is a rapidly progressive and highly lethal infection with mortality rates reaching 100\% if left untreated. However, the transmission route, treatment methods and prognosis have not been well studied.

Case presentation: A 65-year-old male patient presented with a fever, dyspnea, and right upper quadrant abdominal pain. Computed tomography of the chest and abdomen showed huge liver abscess without full liquefaction in the left lobe, large amount of left pleural effusion, and mild pericardial effusion, and the patient was treated with parenteral antibiotics and pigtail insertion at the left pleura. However, four days later, cardiac tamponade was developed and surgical drainage of the abscess and pericardium was performed. Klebsiella pneumonia was isolated from pleural empyema. Twenty-five days after surgery, the patient was discharged without any complications.

Conclusions: Herein, we report a rare case of pleural empyema and pericarditis in that resulted from the extension of huge pyogenic liver abscess. Early surgical treatment may have prevented progression of the pericarditis to the more dismal purulent pericarditis. We also review pertinent English literature on pericarditis as a complication of PLA.
\end{abstract}

Keywords: Liver abscess, Pericarditis, Empyema, Klebsiella pneumoniae

\section{Background}

Pyogenic liver abscess (PLA) is a life-threatening infectious condition, with an estimated incidence of 2.3 cases per 100,000 persons in the United States [1] and 17.6 cases per 100,000 persons in Taiwan [2]. Despite aggressive treatment, the mortality rate ranges from $6 \%$ to $14 \%$ [3]. Klebsiella pneumoniae is an important cause of PLA in Asia, and is often associated with invasive liver abscess syndrome, which accompanies metastatic infection at other sites $[4,5]$. However, pericarditis by direct PLA invasion has rarely been reported [6]. Herein, we report one case of

\footnotetext{
* Correspondence: estevanj@naver.com

${ }^{\dagger}$ Equal contributors

'Department of Internal Medicine, Chonnam National University Hospital,

Gwangju, South Korea

Full list of author information is available at the end of the article
}

a transdiaphragmatic extension of a Klebsiella pneumoniae liver abscess, which caused pleural empyema and pericarditis. We also review pertinent English literature on pericarditis as a complication of PLA.

\section{Case presentation}

A 65-year-old man was transferred to our hospital due to dyspnea. Five days prior to his admission, he had presented to another hospital with right upper quadrant abdominal pain accompanied by fever, and a diagnosis of liver abscess was made. He denied any ophthalmological symptoms such as blurred vision or painful eye swelling. The patient was treated with parenteral ceftriaxone and metronidazole, but his condition deteriorated. On admission, his vital signs were as follows: pulse, 122 beats/min; blood pressure, 
100/60 $\mathrm{mmHg}$; respiratory rate, 24 breaths/min; and temperature, $38.0{ }^{\circ} \mathrm{C}$. On physical examination, decreased breath sounds in the left lower lung fields and tenderness over the right upper abdomen were noted. Laboratory blood tests revealed leukocytosis, elevated liver enzymes (AST $42 \mathrm{U} / \mathrm{L}$, ALT $74 \mathrm{U} / \mathrm{L}$, ALP $162 \mathrm{U} / \mathrm{L}$, and r-GTP $114 \mathrm{U} / \mathrm{L}$ ) and a C-reactive protein level of $18.2 \mathrm{mg} / \mathrm{dL}$. A computed tomography (CT) scan of the abdomen revealed one $10 \times 6 \times 8 \mathrm{~cm}$ and one $4 \times 4 \times 4 \mathrm{~cm}$ liver abscess, without full liquefaction, in the hepatic dome and anterior medial segment, respectively, and a small amount of ascites under the diaphragm. A chest CT revealed a small right pleural effusion, a large left pleural effusion and mild pericardial effusion. A diagnostic thoracentesis was performed. The aspirated pleural fluid was grossly pus $(\mathrm{pH}, 7.009 ; \mathrm{LDH}, 7974 \mathrm{U} / \mathrm{L}$; and protein, $3.3 \mathrm{~g} / \mathrm{dL})$. A pigtail catheter was inserted into the left pleural cavity to drain the empyema. The parenteral ceftriaxone and metronidazole treatment was changed to piperacillin/tazobactam. However, the patient remained febrile and tachypneic and, after four days, he progressed to septic shock. Physical examination revealed jugular venous distension and attenuated heart sounds. The electrocardiogram (ECG) showed multiple premature ventricular complexes. Chest CT revealed an increased volume of the pericardial effusion, with pericardial enhancement (Fig. 1a). Emergent bedside echocardiography showed a moderate amount of pericardial effusion, with some refractile densities in the fluid, suggestive of possible purulent pericarditis. A decision to proceed with surgical drainage of the liver abscess and surgical pericardiostomy was made on post-admission day 5 . The abdominal cavity and liver were examined first.
The huge abscess in the hepatic dome was adherent to the diaphragm. Moreover, the diaphragm and chest wall were inflamed and necrotic, with loculated pus between the diaphragm and the liver abscess (Fig. 1b). The diaphragm was released from the abscess and, after unroofing of the abscess, a drain was inserted into the liver abscess. Subsequently, after partial resection of the xiphoid process, a pericardial window was created (Fig. 1c), and the pericardial fluid, which was serosanguineous, was aspirated. In addition, inflammation of the pericardium was noted and a drain was inserted into the pericardium. After surgical drainage was performed, the patient's condition improved and the ECG normalized. The pleural fluid culture was positive for Klebsiella pneumoniae; however, blood, pericardial fluid and liver abscess cultures were negative. Based on antibiotic susceptibility test, piperacillin/tazobactam was changed to ciprofloxacin. Twenty-five days after surgery, the patient was discharged without any complications. Follow-up abdominal ultrasonography performed at 20 days post-discharge showed a decrease in the size of the liver abscess and subphrenic fluid collection, and complete resolution of the pericardial effusion (Fig. 1d).

\section{Discussion and conclusions}

Acute pericarditis is a rare but potentially lethal complication of a liver abscess, with an incidence rate of $<2 \%$ in patients with an amebic liver abscess [4]. PLA resulting in pericarditis is a rarer occurrence. In our report, we describe a very rare case of a huge liver abscess in the left lobe of the liver that extended transdiaphragmatically, causing a pleural empyema and pericarditis. Although the pleural empyema was successfully managed with insertion of a percutaneous

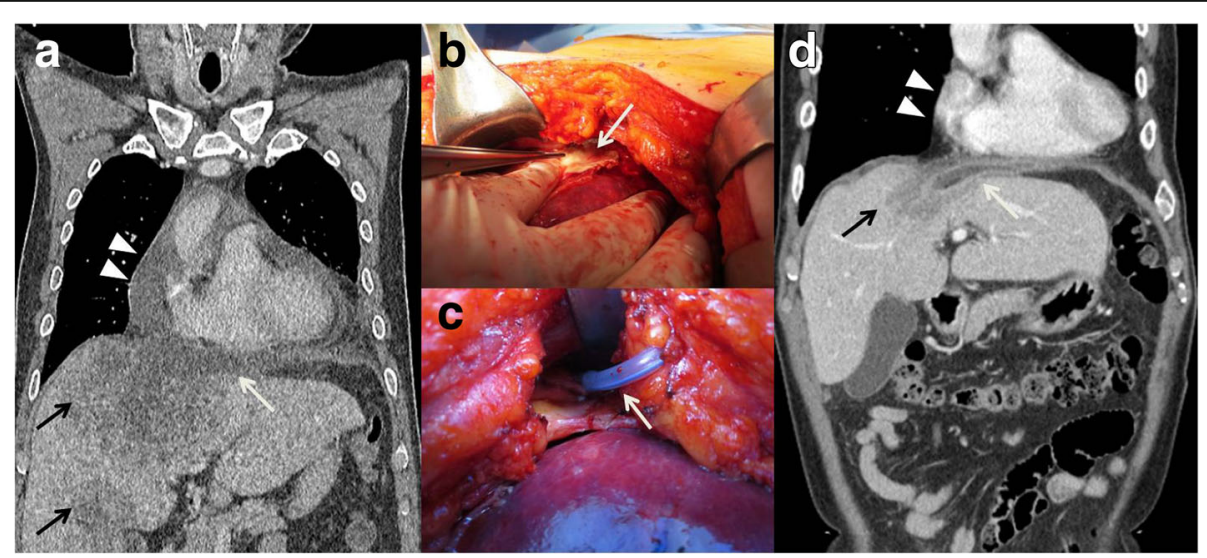

Fig. 1 a. Computed tomography (CT) of chest demonstrated $10 \times 6 \times 8 \mathrm{~cm}$ (segment IV) and $4 \times 4 \times 4 \mathrm{~cm}$ (segment V) sized liver abscesses (black arrows), subphrenic fluid collection (white arrow), and moderate amount of pericaridal effusion (arrowheads). b. Intraoperative findings showed inflamed and necrotic diaphragm (white arrow) abutting liver abscess. c. After pericardial window was created, a pericardial drainage tube was inserted (white arrow). d. Follow-up abdomen CT after 20 days showed decreased size of liver abscess (black arrow) and subphremic fluid collection (white arrow), and complete resolution of pericardial effusion (arrowheads) 
pigtail catheter, the liver abscess could not be initially drained percutaneously as it was not fully liquefied. Despite adequate antibiotic treatment, which was later confirmed to be appropriate for the causative pathogen Klebsiella pneumoniae, pericarditis developed. Inflammation of the diaphragm and pericardium was confirmed during the surgery. Early surgical treatment may have prevented progression of the pericarditis to the more dismal purulent pericarditis.

A PUBMED search of the English literature identified only a few cases of pericarditis as a complication of PLA [6-17], which we have summarized in Table 1. The median age of these patients was 49 years (range, $20 \sim 73$ years), with 8 of the 13 patients (61.5\%) being male. The liver abscess was located in the left lobe of the liver in 10 cases (76.9\%) [6, 7, 9, 11-17] and in the right lobe (segment V/VIII) in one case (7.7\%) [8], with involvement of both right and left lobes in one case $(7.7 \%)$ [10]. In our case, the abscess was located in the left lobe. The transmission route of the PLA to the pericarditis was transdiaphragmatic in most of the previously reported cases [6, 10-15, 17], including our case.

In previously reported cases of PLA with pericarditis, patients complained of various symptoms including a fever, abdominal pain, dyspnea, and chest pain [6-17]. Tachycardia and septic shock were usually present. Distention of the jugular veins or an elevated jugular pressure are the most important finding suggestive of a pericardial involvement, due to the development of septic shock, which usually causes the central venous pressure to fall and the jugular veins to collapse. This finding was positive in twelve patients $(92.3 \%)$ [6, 8-17], and in our case as well. All of these patients developed a cardiac tamponade that can be fatal if left untreated. Therefore, early identification is crucial. However, ECG findings in these cases can vary, including ST segment elevations $[6,7,17]$, low voltage in limb leads [12] and ventricular tachycardia [14], or the ECG can be normal [15]. In our case, the ECG showed multiple premature ventricular complexes. An enlarged cardiac silhouette is frequently observed on chest X-rays $[6,11,14,16,17]$, with or without pleural effusion. On CT imaging of the abdomen or chest, liver abscesses with pericardial effusion are a frequent finding $[6,8-10,12-15]$. Therefore, if patients with liver abscess complain of dyspnea or chest pain and distended jugular veins are noted, a thorough evaluation, including chest $\mathrm{CT}$ or transthoracic echocardiography, for pericardial involvement is needed.

In terms of causative pathogen for PLA associated with pericarditis, $K$. pneumoniae was identified in four cases $(30.7 \%)[6,12,14]$, E.coli in four cases (30.7\%) [8, $11]$ and Actinomyces spp. $[9,10]$ in two cases $(15.4 \%)$. Other responsible pathogens previously reported include
M. morganii [16], $K$. oxytoca [10], C. koseri [10], Candida [6], Enterococcus [8, 15], Fusobacterium [7, 9], Peptostreptococcus [9], and Proteus [15]. Although various pathogens have been described for the cause of PLA, $K$. pneumoniae is the most common responsible pathogen in Asia [5]. Jun et al. have reported K. pneumoniae as the most commonly isolated organism in the abscess $(49.8 \%)$, followed by Streptococcus species (3.8\%) and E. coli (3.3\%). K. pneumoniae was also the most commonly isolated organism in blood (70.2\%), followed by E. coli (10.7\%) [18]. However, the most common pathogen associated with bacterial pericarditis is not $K$. pneumonia. In one study involving 933 acute pericarditis patients, 32 patients had bacterial origin, and staphylococci $(21.9 \%)$ and streptococci $(15.6 \%)$ were the most common organisms. K. pneumoniae was responsible for only $3.1 \%$ of cases [19]. It is very interesting that $K$. pneumoniae was the most common pathogen for PLA associated with pericarditis including our case.

It has been reported that K1 strains of $K$. pneumoniae are associated with PLA complicated by metastatic endophthalmitis or CNS infections [20], and $\mathrm{Yu}$ et al. have reported a case of bacterial pericarditis by genotype K1 [21]. Endophthalmitis is the most common metastatic complication of PLA, and the incidence is reported to be $0.84-1.92 \%$. Symptoms of endophthalmitis are visual impairment leading to blindness, or painful eye swelling. Suggested risk factors of endophthalmitis include diabetes mellitus, K1 strains of $K$. pneumoniae, abscess in the right superior segment, and other systemic infection. Intravitreal antibiotics and early vitrectomy are the mainstay of the treatment, but the prognosis is poor [4].

The K1 strain has been reported to be highly virulent because of hypermucoviscosity, which is associated with high serum resistance, high-level resistance to phagocytosis, and resistance to complement deposition [20, 21]. Although the genotypic analysis has not been performed in the reported cases, it is possible that highly virulent strains of $K$. pneumoniae were associated with PLA complicated by pericarditis. Thus, further studies are needed to identify virulent strains that are responsible for PLA associated with pericarditis.

Among the 12 cases reporting purulent pericarditis as a complication of PLA, pericardial fluid culture was positive in all patients $[6,8,9,11-17]$. In contrast, the pericardial fluid in our case did not reveal any microorganism. This is probably because early detection and surgical treatment of pericarditis prevented progression to purulent pericarditis. Although PLA was the primary infection focus, only eight cases $(61.5 \%)$ reported positive cultures of hepatic abscess [7-9, 12-14, 16]. Rates of positive blood cultures were even lower, with only four $(30.8 \%)$ reported cases [7, 13, 16, 17]. Both hepatic 


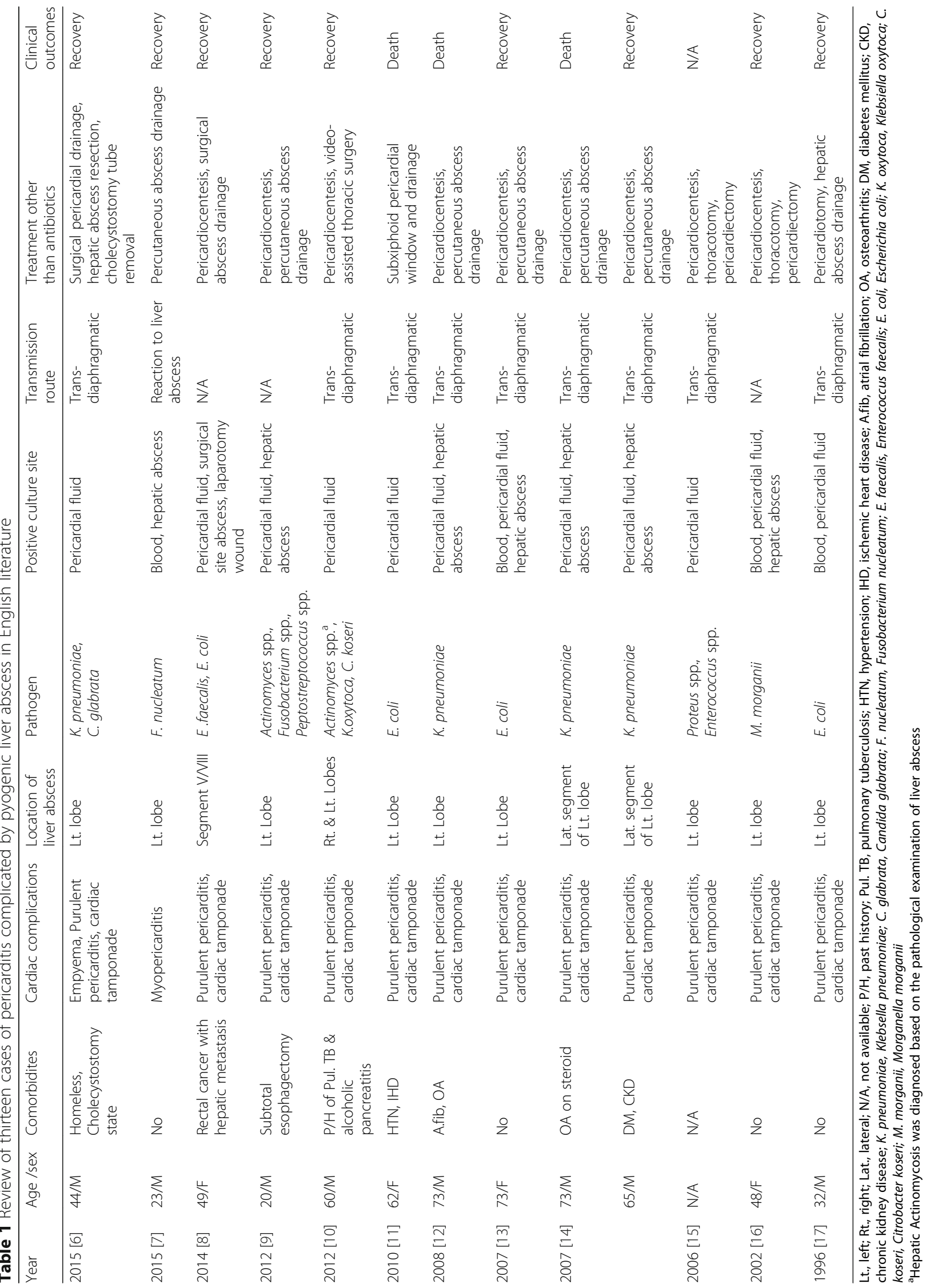


abscess and blood culture results were negative in our case. The negative culture from hepatic abscess may be due to the delay in obtaining the culture (nine days after initial antibiotic treatment) as the initial percutaneous aspiration was not possible. However, the negative blood culture is noteworthy in light of such an overwhelming infection, with positive blood cultures typically reported in $50 \%$ of cases of PLA [22].

The following treatments for PLA-associated pericarditis have been reported: pericardiocentesis in six cases (46.1\%) [8, 9, 12-14]; surgical pericardial drainage or pericardiectomy in six cases $(46.1 \%)[6,10,11,15-17]$, combined with percutaneous abscess drainage in seven cases $(53.8 \%)$ [7, 9, 12-14, 17]; and surgical abscess resection in two cases $(15.4 \%)[6,8]$. All patients received antibiotic treatments. Three patients $(23 \%)$ died among the previously reported cases, indicative of the higher mortality rate for PLA-associated pericarditis than the reported mortality rate of $5-10 \%$ for PLA alone [23].

In conclusion, pericarditis is a rare but fatal complication of left lobe liver abscess. Pericarditis should be highly suspected in patients with cardiac symptoms, jugular vein engorgements and enlarged cardiac silhouette on chest Xray. Early diagnostic evaluation, using chest $\mathrm{CT}$ or transthoracic echocardiography, and immediate treatment, including pericardiocentesis or pericardiotomy and abscess drainage, combined with intravenous antibiotics can lower the risk of patient death.

\section{Abbreviations \\ ALP: Alkaline phosphatase; ALT: Alanine Aminotransferase; AST: Aspartate Aminotransferase; C. glabrata: Candida glabrata; C. koseri: Citrobacter koseri; CT: Computed tomography; E. coli: Escherichia coli; E. faecalis: Enterococcus faecalis; ECG: Electrocardiogram; F. nucleatum: Fusobacterium nucleatum; $K$. oxytoca: Klebsiella oxytoca; K. pneumonia: Klebsella pneumoniae; LDH: Lactate dehydrogenase; M. morganii: Morganella morganii; PLA: Pyogenic liver abscess; r-GTP: Gamma Glutamyl Transpeptidase}

\section{Acknowledgements}

None.

\section{Funding}

None.

\section{Availability of data and materials}

The data used in the current study are available from the corresponding author on reasonable request.

\section{Authors' contributions}

EC, SWP designed the study and wrote the manuscript. CHJ participated in the design of the study and the study's coordination. SSS contributed to the imaging and interpretation. EKP and KSL performed surgical treatment. SYP managed the patient and helped to draft the manuscript. CHP, HSK acquired and analyzed the laboratory and clinical data. SKC, JSR participated in reviewing the literature and editing the initial manuscript. All authors read and approved the final manuscript.

Ethics approval and consent to participate Not applicable.

\section{Consent for publication}

Written informed consent was obtained from the patient for publication of this case report.

\section{Competing interests}

The authors declare that they have no competing interests.

\section{Publisher's Note}

Springer Nature remains neutral with regard to jurisdictional claims in published maps and institutional affiliations.

\section{Author details}

${ }^{1}$ Department of Internal Medicine, Chonnam National University Hospital, Gwangju, South Korea. ${ }^{2}$ Department of Radiology, Chonnam National University Hospital, Gwangju, South Korea. ${ }^{3}$ Department of Surgery, Chonnam National University Medical School, Gwangju, South Korea. ${ }^{4}$ Department of Thoracic and Cardiovascular Surgery, Chonnam National University Hospital, Gwangju, South Korea.

Received: 9 August 2017 Accepted: 8 January 2018

Published online: 15 January 2018

\section{References}

1. Kaplan GG, Gregson DB, Laupland KB. Population-based study of the epidemiology of and the risk factors for pyogenic liver abscess. Clin Gastroenterol Hepatol. 2004;2(11):1032-8.

2. Tsai FC, Huang YT, Chang LY, Wang JT. Pyogenic liver abscess as endemic disease, Taiwan. Emerg Infect Dis. 2008;14(10):1592-600.

3. Alvarez Perez JA, Gonzalez JJ, Baldonedo RF, Sanz L, Carreno G, Junco A Rodriguez Jl, Martinez MD, Jorge Jl. Clinical course, treatment, and multivariate analysis of risk factors for pyogenic liver abscess. Am J Surg. 2001;181(2):177-86.

4. Park IH, Jun CH, Wi JW, Park SY, Lee WS, Jung SI, Park CH, Joo YE, Kim HS, Choi SK, et al. Prevalence of and risk factors for endogenous endophthalmitis in patients with pyogenic liver abscesses. Korean J Intern Med. 2015;30(4):453-9

5. Wang JH, Liu YC, Lee SS, Yen MY, Chen YS, Wang JH, Wann SR, Lin HH. Primary liver abscess due to Klebsiella Pneumoniae in Taiwan. Clin Infect Dis. 1998:26(6):1434-8.

6. Reddy G, Chatterjee A, Brott BC. Transdiaphragmatic rupture of hepatic abscess producing purulent pericarditis and pericardial tamponade. Circulation. 2015:131(1):e1-2

7. Kearney A, Knoll B. Myopericarditis associated with Fusobacterium nucleatum-caused liver abscess. Infect Dis (Lond). 2015:47(3):187-9.

8. Fidalgo Garcia M, Rodriguez Sanjuan JC, Riano Molleda M, Gonzalez Andaluz M, Real Noval H, Gomez Fleitas M. Purulent pericarditis after liver abscess: a case report. Case Rep Med. 2014;2014:735478.

9. Llenas-Garcia J, Lalueza-Blanco A, Fernandez-Ruiz M, Villar-Silva J, Ochoa M, Lozano F, Lizasoain M, Aguado JM. Primary hepatic actinomycosis presenting as purulent pericarditis with cardiac tamponade. Infection. 2012; 40(3):339-41.

10. Sakaguchi Y, Isowa N, Nakazaki H, Takeda K, Tokuyasu H, Saitoh Y, Soeda T, Ohe T, Tokuyasu Y, Miura H. Acute cardiac tamponade caused by the extension of multiple hepatic actinomycotic abscesses. Intern Med. 2012:51(3):305-8.

11. Chong VH, Zainal-Abidin Z, Hassan H, Chong CF. Rare complications of pyogenic liver abscess. Singap Med J. 2010:51(10):e169-72.

12. Hara N, Kato M, Fuse K, Nagata T, Kimura Y, Sugiura H, Saito A, Fujita S, Ikeda Y, Kitazawa $\mathrm{H}$, et al. A rare case of pyogenic liver abscess complicated with cardiac tamponade. Intern Med. 2008;47(6):563-4.

13. Wu CC, Hsu TF. An unusual cause of shock in a patient with liver abscess. Int J Cardiol. 2007:115(3):e114-5.

14. Vong SC, Guo HR, Lin HJ, Foo NP. Cardiac tamponade secondary to pyogenic liver abscess. J Clin Gastroenterol. 2007;41(6):635-6.

15. Kumar D, Zaidi SM, Jenkins PF. Intra-pericardial rupture of bacterial hepatic abscess: an unusual cause of 'cardiac' chest pain. Acute Med. 2006;5(3):96-8.

16. Tsai WC, Chang LK. Morganella Morganii causing solitary liver abscess complicated by pyopericardium and left pleural effusion in a nondiabetic patient. J Microbiol Immunol Infect. 2002;35(3):191-4. 
17. Cho MC, Kim DW, Earm JH, Yoon SJ, Kim ST, Hong JM, Ahn JH, Hong JS. Pericardial abscess due to transdiaphragmatic perforation of the pyogenic liver abscess. Eur Heart J. 1996;17(10):1602-3.

18. Jun $\mathrm{CH}$, Yoon JH, Wi JW, Park SY, Lee WS, Jung SI, Park CH, Joo YE, Kim HS, Choi SK, et al. Risk factors and clinical outcomes for spontaneous rupture of pyogenic liver abscess. J Dig Dis. 2015;16(1):31-6.

19. Gouriet F, Levy PY, Casalta JP, Zandotti C, Collart F, Lepidi H, Cautela J, Bonnet JL, Thuny F, Habib G, et al. Etiology of Pericarditis in a prospective cohort of 1162 cases. Am J Med. 2015;128(7):784. e781-788

20. Fang CT, Lai SY, Yi WC, Hsueh PR, Liu KL, Chang SC. Klebsiella Pneumoniae genotype K1: an emerging pathogen that causes septic ocular or central nervous system complications from pyogenic liver abscess. Clin Infect Dis. 2007:45(3):284-93.

21. Yu WL, Cheng CC, Chuang YC. First report of acute purulent pericarditis by capsule genotype K1 Klebsiella Pneumoniae in an alcoholic patient. Diagn Microbiol Infect Dis. 2009;63(3):346-7.

22. Chemaly RF, Hall GS, Keys TF, Procop GW. Microbiology of liver abscesses and the predictive value of abscess gram stain and associated blood cultures. Diagn Microbiol Infect Dis. 2003;46(4):245-8.

23. Chen SC, Huang CC, Tsai SJ, Yen CH, Lin DB, Wang PH, Chen CC, Lee MC. Severity of disease as main predictor for mortality in patients with pyogenic liver abscess. Am J Surg. 2009;198(2):164-72.

Submit your next manuscript to BioMed Central and we will help you at every step:

- We accept pre-submission inquiries

- Our selector tool helps you to find the most relevant journal

- We provide round the clock customer support

- Convenient online submission

- Thorough peer review

- Inclusion in PubMed and all major indexing services

- Maximum visibility for your research

Submit your manuscript at www.biomedcentral.com/submit
Biomed Central 